\title{
VIGENCIA ACTUAL DE LOS NOMOGRAMAS EN LA ESTADIFICACIÓN DEL CÁNCER DE PRÓSTATA.
}

\author{
Ángel Borque Fernando, Gerardo Sanz? , Luís Mariano Esteban², María Jesús Gil Sanz, Pedro \\ Gil Martínez, Ángel García de Jalón, Patricia Serrano Frago, Carlos Allepuz Losa y Luís Ángel \\ Rioja Sanz.
}

Servicio de Urología. Hospital Universitario Miguel Servet. Zaragoza.

'Departamento de Métodos Estadísticos. Facultad de Ciencias. Universidad de Zaragoza.

${ }^{2}$ Departamento de Matemática Aplicada. Escuela Universitaria Politécnica La Almunia de Doña Godina. Universidad de Zaragoza. España.

\begin{abstract}
Resumen.- OBJETIVO: En este trabajo se presenta una revisión del concepto de "nomograma" aplicado al cáncer de próstata y específicamente como medio de estadificación.

MÉTODOS/RESULTADOS: Para ello se describen los parámetros indispensables para evaluar este tipo de modelos predictivos, a saber: Calibración, Discriminación y Utilidad Clínica. Estos requisitos son analizados sobre un caso práctico real en nuestro medio asistencial comparando las "Tablas de Partin" y el "Nomograma del Hospital Universitario Miguel Servet", demostrando su correcta calibración, discriminación y utilidad clínica previa selección de adecuados puntos de corte.
\end{abstract}

CONCLUSIÓN: La aplicación del modelo predictivo a nuestra práctica asistencial ha logrado una infraestadificación clínica tras prostatectomía radical del 17,3\%.

Palabras clave: Nomogramas. Cáncer de próstata. Estadificación.
Summary.- OBJETIVE: This paper presents a review of the concept of "nomogram" applied to prostate cancer, and specifically as a staging tool.

METHODS/RESULTS: We describe the essential parameters for the evaluation of such type of predictive models: Calibration, discrimination and clinical usefulness. Such requisites are analyzed using a real clinical case in our clinical setting, comparing the "Partin's tables" and the "Miguel Servet University Hospital's nomogram". We demonstrate its correct calibration, discrimination and clinical usefulness after previous selection of proper cut points.

CONCLUSION: The application of the predictive model to our clinical practice has achieved a clinical understaging of $17.3 \%$ after radical prostatectomy.

Keywords: Nomograms. Prostate cancer. Staging.

\section{INTRODUCCIÓN}

\section{"Representación gráfica que permite realizar con ra- pidez cálculos numéricos aproximados."}

Ésta no es sino la definición del vocablo "nomograma" y sin duda alguna precisa su verdadero significado.

Cuando recurrimos a un nomograma el trasfondo de nuestro problema ha sido resuelto mediante una función matemática integrada previsiblemente por múltiples variables o factores pronósticos, o incluso pocos o uno sólo pero que en cualquier caso son ponderados por un desarrollo matemático complejo que hace laboriosa su aplicabilidad práctica y en consecuencia, especialmente expuesta a error. 
Veamos algún ejemplo práctico que nos ayude a entender mejor este concepto:

Si quisiéramos conocer la superficie corporal de uno de nuestros pacientes podemos aplicar la fórmula descrita por D. Dubois (1):

Superficie corporal $\left(\mathrm{m}^{2}\right)=$ Peso $(\mathrm{Kg})^{0,425} \times$ Altura $\left(\mathrm{cm}^{0,725} \times 0,007184\right.$

Lo cual supone cuando menos un cálculo matemático si no complejo, si al menos laborioso y en cualquier caso susceptible de error.

Sin embargo, cabe recurrir a la representación gráfica de éste cálculo (Gráfico 1) en la que de un modo sin duda alguna mucho más rápido alcanzaríamos un valor numérico muy aproximado al obtenido al aplicar la fórmula y en cualquier caso menos susceptible de error.

Si quisiéramos conocer la probabilidad de que un tumor prostático se encuentre patológicamente órgano-confinado (pT2) tras la prostatecto- mía, podemos recurrir a la siguiente fórmula matemática (2):

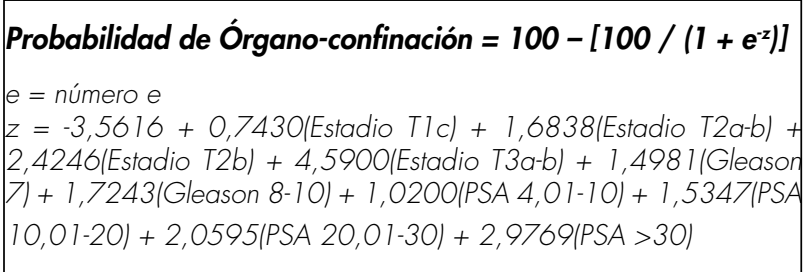

O bien utilizar la Tabla I ladaptado de Borque y cols. ${ }^{2}$ ) en la que de un modo infinitamente más sencillo podríamos obtener un resultado muy aproximado, y sin duda con menos posibilidad de error.

En ambos ejemplos no hemos hecho sino recurrir a una representación gráfica, bien sea en forma literal de gráfico o bien como una tabla, que nos ha permitido realizar con rapidez cálculos numéricos aproximados, quizás menos precisos que el uso de la fórmula planteada pero de seguro infinitamente más rápidos y menos sujetos a error; hemos recurrido pues a un nomograma.

TABLA I. PROBABILIDAD DE ÓRGANO-CONFINACIÓN TUMORAL (pT2) - T.N.M. 2002

\begin{tabular}{|c|c|c|c|c|c|}
\hline PSA preop. (ng./mL.) & \multirow[t]{2}{*}{$0-4$} & \multirow[t]{2}{*}{$4,01-10$} & \multirow[t]{2}{*}{$10,01-20$} & \multirow[t]{2}{*}{$20,01-30$} & \multirow[t]{2}{*}{$>30$} \\
\hline Estadio clínico & & & & & \\
\hline \multicolumn{6}{|c|}{ Suma de Gleason de la biopsia: 2 - 6} \\
\hline T1a-T1b & 97 & 93 & 88 & 82 & 64 \\
\hline Tlc & 94 & 86 & 78 & 68 & 46 \\
\hline $\mathrm{T} 2 \mathrm{a}-\mathrm{T} 2 \mathrm{~b}$ & 87 & 70 & 58 & 45 & 25 \\
\hline $\mathrm{T} 2 \mathrm{c}$ & 76 & 53 & 40 & 28 & 14 \\
\hline$T 3 a-T 3 b$ & 26 & 11 & 7 & 4 & 2 \\
\hline \multicolumn{6}{|c|}{ Suma de Gleason de la biopsia: 7} \\
\hline T1a-T1b & 89 & 74 & 63 & 50 & 29 \\
\hline Tlc & 79 & 57 & 45 & 32 & 16 \\
\hline $\mathrm{T} 2 \mathrm{a}-\mathrm{T} 2 \mathrm{~b}$ & 59 & 35 & 23 & 16 & 7 \\
\hline $\mathrm{T} 2 \mathrm{c}$ & 41 & 20 & 13 & 8 & 3 \\
\hline$T 3 a-T 3 b$ & 7 & 3 & 2 & 1 & 0 \\
\hline \multicolumn{6}{|c|}{ Suma de Gleason de la biopsia: 8 - 10} \\
\hline T1a-T1b & 86 & 69 & 58 & 44 & 24 \\
\hline Tlc & 75 & 52 & 39 & 28 & 13 \\
\hline $\mathrm{T} 2 \mathrm{a}-\mathrm{T} 2 \mathrm{~b}$ & 54 & 30 & 20 & 13 & 6 \\
\hline $\mathrm{T} 2 \mathrm{c}$ & 36 & 17 & 11 & 7 & 3 \\
\hline T3a - T3b & 6 & 2 & 1 & 1 & 0 \\
\hline
\end{tabular}



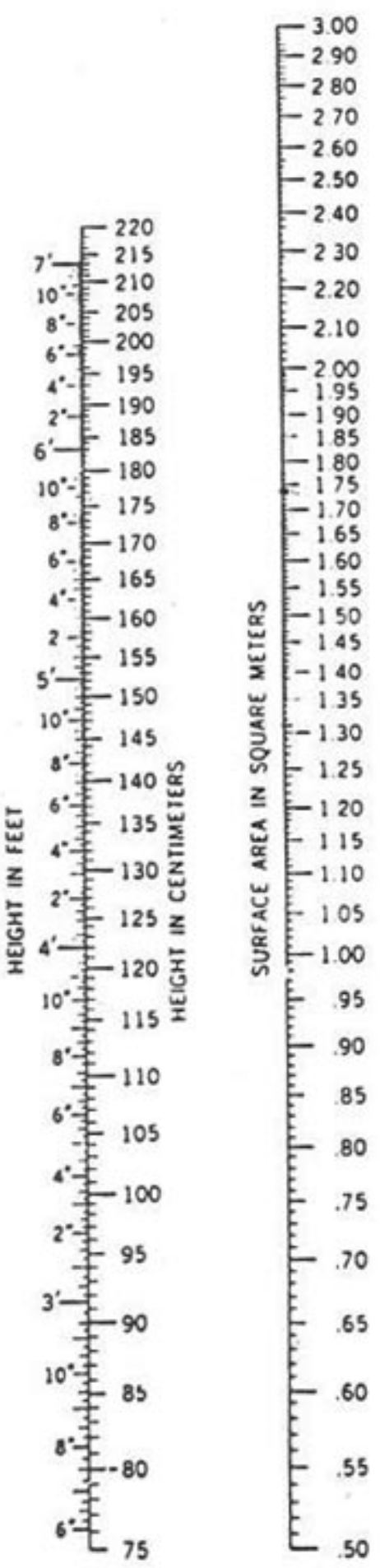

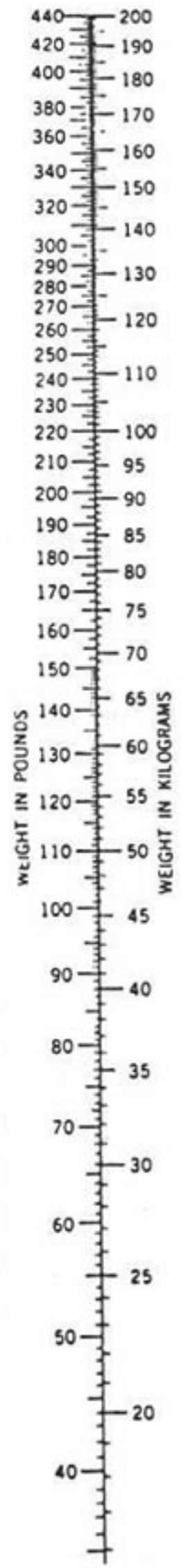

GRÁFICO 1.
Esclarecido pues este primer aspecto conceptual acerca de lo que es un nomograma, resulta fácil comprender que en general estos modelos gráficos esconden tras de sí un complejo análisis multivariante de difícil aplicabilidad y comprensión en su desarrollo matemático.

Las limitaciones prácticas para su aplicabilidad quedan resueltas con el diseño del nomograma, pero la comprensión del modelo matemático que lo genera no se resuelve con ese diseño gráfico simplificado. De hecho en no pocas ocasiones se presenta directamente el nomograma (3-7) diseñado sin explicitarse la fórmula matemática que lo ha generado, todo ello hace que en general sean concebidos como una herramienta interesante e incluso atractiva por lo novedosa pero de génesis incierta o reservada para "unos pocos fanáticos de la estadística" lo que además de ser incierto, genera desconfianza en su uso y sin duda alguna limita su difusión y estandarización.

\section{NOMOGRAMAS EN CÁNCER DE PRÓSTATA}

Son infinidad los nomogramas que se han diseñado para su aplicación en cáncer de próstata, con un crecimiento exponencial en los últimos años. Podríamos clasificarlos en tres grandes grupos según su propósito:

Nomogramas diagnósticos: entendemos por tales aquellos modelos predictivos de análisis multivariante que han pretendido estimar la probabilidad de que un determinado paciente padeciera cáncer de próstata (8-16) o quizás el más conocido como nomograma de Viena (17) que pretende precisar el número de cilindros a tomar para obtener una mayor rentabilidad diagnóstica de la biopsia.

Nomogramas de estadificación: en este campo hemos de citar por derecho propio el modelo predictivo conocido como "Tablas de Partin" tanto en su desarrollo inicial y más difundido del año 1997 (3), como en su posterior actualización del año 2001 (4). Pero estos trabajos, aún siendo los más conocidos no son ni mucho menos los únicos tal y como recogía una excelente revisión de Ross y cols. (18). que ya ha quedado algo trasnochada en el tiempo por la posterior aparición de múltiples nomogramas predictivos del estadio patológico con posterioridad (19-22) y en la que se analizaban detalladamente 18 nomogramas predictivos del estadio patológico, el primero de ellos del año 1987, con diferente éxito. También a nivel nacional hemos tenido la oportunidad de desarrollar un modelo predictivo multivariante de órganoconfinación tumoral en cáncer de próstata (2). 
Nomogramas pronósticos: y finalmente hemos de mencionar el grupo más reciente de este tipo de modelos predictivos en cáncer de próstata que es el referente a la estimación del resultado de la aplicación de diferentes tratamientos tras el diagnóstico de adenocarcinoma de próstata. Sin duda alguna el máximo representante de estos modelos predictivos es M. Kattan quien ya en 1998 publicó su primer nomograma sobre la evolución tras prostatectomía radical (23). Como cabía esperar el mayor número de nomogramas predictivos de la evolución tras tratamiento del cáncer de próstata son los que evalúan el resultado tras prostatectomía radical $(5,24-35)$ pero también los hay que estiman la progresión tras radioterapia externa $(6,36-38)$ o braquiterapia (7), o incluso tras castración (39) o situación de andrógenoindependencia (40).

\section{VIGENCIA ACTUAL DE LOS NOMOGRAMAS EN LA ESTADIFICACIÓN DEL CÁNCER DE PRÓSTATA}

Sin embargo, como es obvio no podemos abordar un análisis pormenorizado de cada uno de estos nomogramas anteriormente detallados, que muy posiblemente ni tan siquiera son todos los publicados, sino que vamos a entrar a analizar uno de ellos que por otro lado es el posiblemente más conocido, las "Tablas de Partin", en un intento de proporcionar al lector las herramientas para evaluar estos modelos predictivos y tras ello ganando su confianza si así lo merecen, pasar a integrarlo como una herramienta más de su armamentario diagnóstico-terapéutico; pues es innegable que aunque la inmensa mayoría

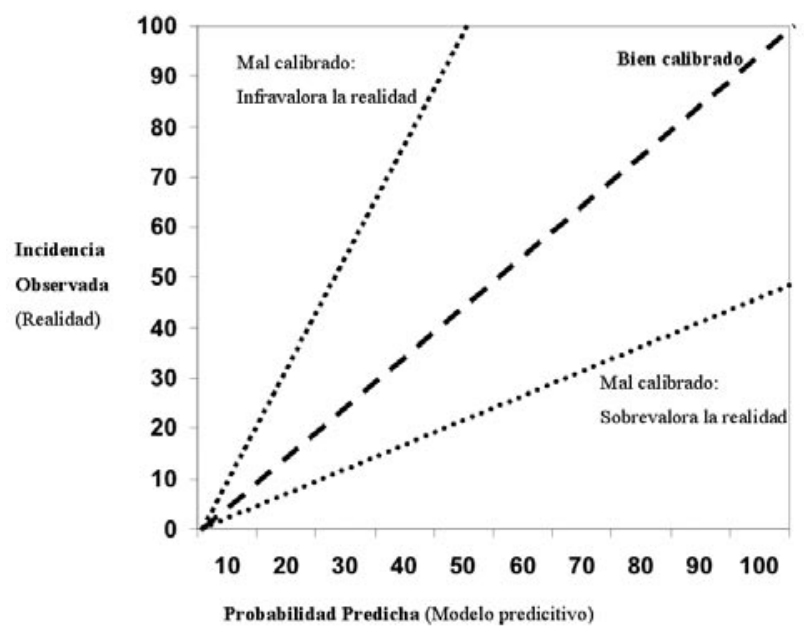

FIGURA 1. Esquema interpretativo sobre las curvas de calibración. de los urólogos conoce de su existencia y modo de aplicación, son muy pocos los que lo utilizan como una herramienta de ayuda en la toma de decisiones individualizadas con sus pacientes.

Dos excelentes publicaciones marcan a nuestro juicio la pauta acerca de cómo validar este tipo de modelos predictivos de análisis multivariante, una primera de carácter más general publicada en 2002 por Vergouwe y cols. (41), y una segunda más específica sobre cáncer de próstata aparecida al año siguiente de Di Blasio y cols. (42).

En síntesis podríamos considerar que estos modelos predictivos deben cumplir tres requisitos que son:

Calibración: Entendiendo por tal el conocer si hay una buena correlación entre los resultados predichos y lo que me encuentro en mi medio. Es decir, que si el modelo predice que en un contexto determinado un resultado ocurre en un $80 \%$ de los casos, que en dicho contexto y en la realidad de mi práctica asistencial ese resultado realmente se dé en 8 de cada 10 casos.

La forma más gráfica y visual de estimar la calibración es mediante curvas de calibración en las que las probabilidades predichas se suelen representar en el eje de abscisas y las incidencia real observada en nuestro medio se suele representar en el eje de ordenadas (Figura 1). De este modo la situación ideal correspondería a una recta que discurriera por la bisectriz

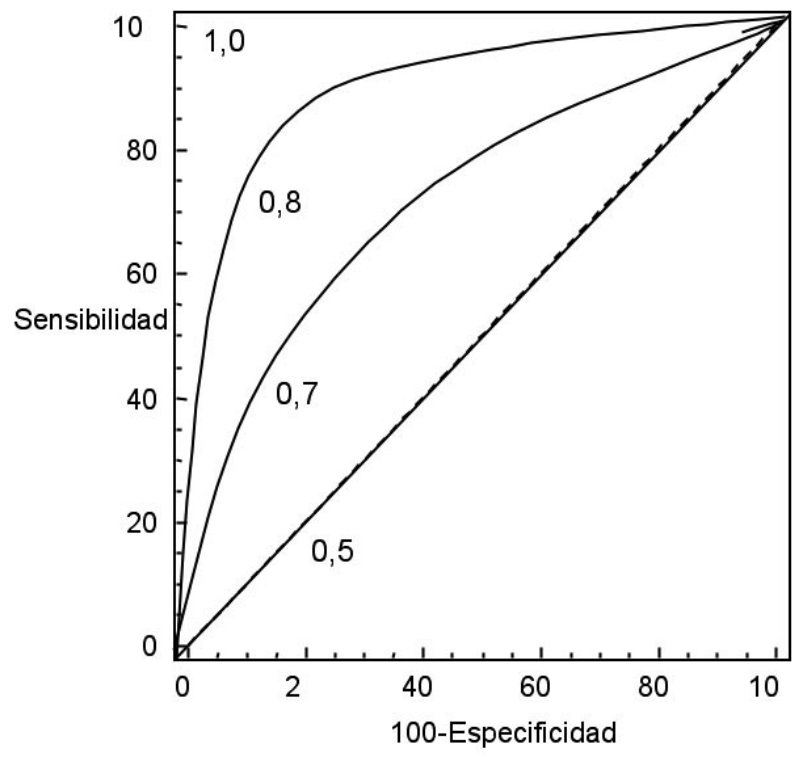

FIGURA 2. Esquema interpretativo sobre las áreas bajo la curva ROC (discriminación). 
del ángulo del eje de coordenadas de modo que probabilidades predichas e incidencias observadas coincidieran, toda recta/curva que discurra por el área superior a esta bisectriz supondrá una infravaloración en tanto que las probabilidades predichas serán inferiores a la incidencia observada en la realidad, y toda recta/curva que discurra por el área inferior a la bisectriz corresponderá a una sobrevaloración del modelo en el que sus probabilidades predichas serán superiores a la realidad observada.

Discriminación: Que hace referencia a constatar si el modelo predictivo es capaz de distinguir entre los "casos" y los "no casos". Es decir, que ya no sólo sea capaz de expresarnos un porcentaje de que algo ocurra o no, sino que sea capaz en base a ese porcentaje de distinguir entre los casos en que ese algo acontezca y los que no.

La capacidad de discriminación de un modelo diagnóstico se suele precisar mediante curvas de rendimiento diagnóstico o más conocidas como curvas ROC (Receiver Operating Characteristics), y concretamente mediante las áreas bajo la curva ROC (ABC-ROC). Así una ABC-ROC de 0,50 (50\%) equivale a una capacidad de discriminación nula del modelo predictivo teniendo la misma valía que lanzar una moneda al aire y esperar a que el cara o cruz de la moneda (probabilidad de que ocurra una u otra opción: $50 \%$ ), nos diera el resultado. Cuando la $A B C-R O C$ llega al 0,70 (70\%) se considera como aceptable la capacidad de discriminación del modelo; es buena si es del 0,80 (80\%); y obviamente perfecta cuando llega al 1,0 (100\%) (Figura 2).

Utilidad clínica: Que no es sino precisar cómo el nomograma mejora mi práctica clínica habitual.

Este aspecto no es sino conocer los "puntos de corte" óptimos a partir de los parámetros de validez externa (sensibilidad, especificidad y valores predictivos positivo y negativo) obtenidos en mi medio y que me permiten aplicarlo a mi trabajo asistencial en la toma de decisiones.

\section{Validación externa de las Tablas de Partin}

Llegados a este punto y establecidas estas premisas, existen en nuestro conocimiento seis validaciones externas publicadas de las "Tablas de Partin" (43-48) y una séptima desarrollada por nosotros en nuestro medio y pendiente de publicación que compara el modelo de Partin y cols. (4) con el desarrollado por nosotros (2).

Veamos cómo en cada una de estas seis validaciones publicadas se analizan los tres criterios an- teriormente citados centrándonos en la estimación de la probabilidad de órgano-confinación tumoral (estadio pT2), para no hacer esta revisión excesivamente extensa:

Blute y cols. (43) publican en el año 2000 la primera validación externa de las "Tablas de Partin" en su modelo de 1997 (3). El modelo fue aplicado a 2.475 pacientes tratados en la Clínica Mayo (U.S.A.) objetivando una infravaloración de las "Tablas de Partin" con respecto a la realidad de su medio en lo que a calibración se refiere, así como una ABC-ROC de 0,727 lo que le confería una capacidad discriminativa aceptable. La utilidad clínica no fue analizada.

Dos años más tarde, Penson y cols. (44) aplica igualmente el modelo de Partin de 1997 (3) a 1.162 pacientes de la base de datos CaPSURE (U.S.A.). En este caso ni calibración ni utilidad clínica fueron analizadas y la capacidad de discriminación fue mínimamente aceptable con una $A B C-R O C$ de 0,684 .

En 2003, Graefen y cols. (45) aplican nuevamente las "Tablas de Partin" de 1997 (3) a 1.131 pacientes europeos tratados en la Universidad de Hamburgo (Alemania). Tampoco se analizan calibración ni utilidad clínica, y la discriminación es buena con una $A B C-R O C$ de 0,817 (I.C. 95\%: 0,757-0,856).

Al año siguiente, este mismo grupo en un trabajo firmado por Augustin y cols. (46) comparan las "Tablas de Partin" de 1997 (3) y su posterior actualización de 2001 (4) sobre 2.139 pacientes de la Universidad de Hamburgo (Alemania), nuevamente. En ambos casos se obtienen buenas curvas de calibración, quizás algo mejores en el modelo del 2001 ; y en cuanto a la discriminación es buena con valores de ABC-ROC de 0,784 (I.C. 95\%: 0,7640,805 ) para el modelo de 1997 y de 0,787 (I.C. 95\%: 0,766-0,807) para el del 2001. Una vez más la utilidad clínica no se evaluó.

El mismo grupo en el 2005, en manos de Steuber y cols. (47) aplica a 1.990 pacientes lógicamente de la Universidad de Hamburgo el modelo del 2001 (4) y obtiene una buena curva de calibración así como buena capacidad de discriminación con una ABC-ROC de 0,79 (I.C. 95\%: 0,77-0,82). Como en los casos anteriores la utilidad clínica no se detalla.

$Y$ finalmente en el mismo año 2005, Eskicorapci y cols. (48) sobre 1.043 pacientes procedentes de 13 hospitales de Turquía, encuentra una escasamente aceptable capacidad de discriminación del modelo del 2001 (4) con una ABC-ROC de 0,666. 
Calibración y utilidad clínica, nuevamente no fueron evaluadas.

Con todo lo hasta ahora expuesto podríamos concluir que en general en los escasos trabajos en que se evalúa la calibración de las "Tablas de Partin", ésta es buena $(46,47)$ salvo en el caso de la Clínica Mayo como consecuencia del diferente manejo anatomo-patológico de las piezas de prostatectomía

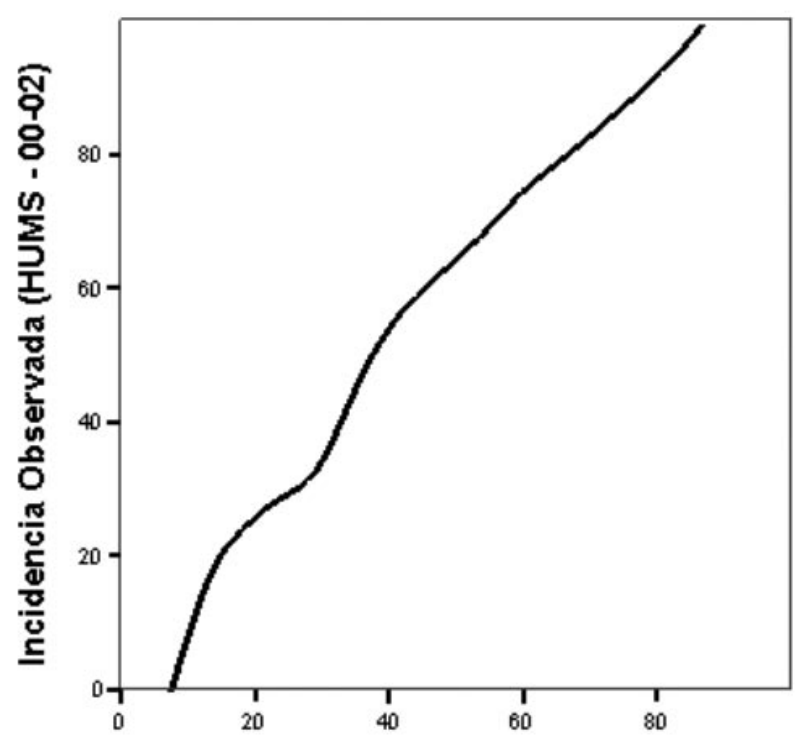

Probabilidad Predicha (Partin - 2001)

FIGURA 3. Curva de calibración de las "Tablas de Partin" aplicadas a nuestro medio.

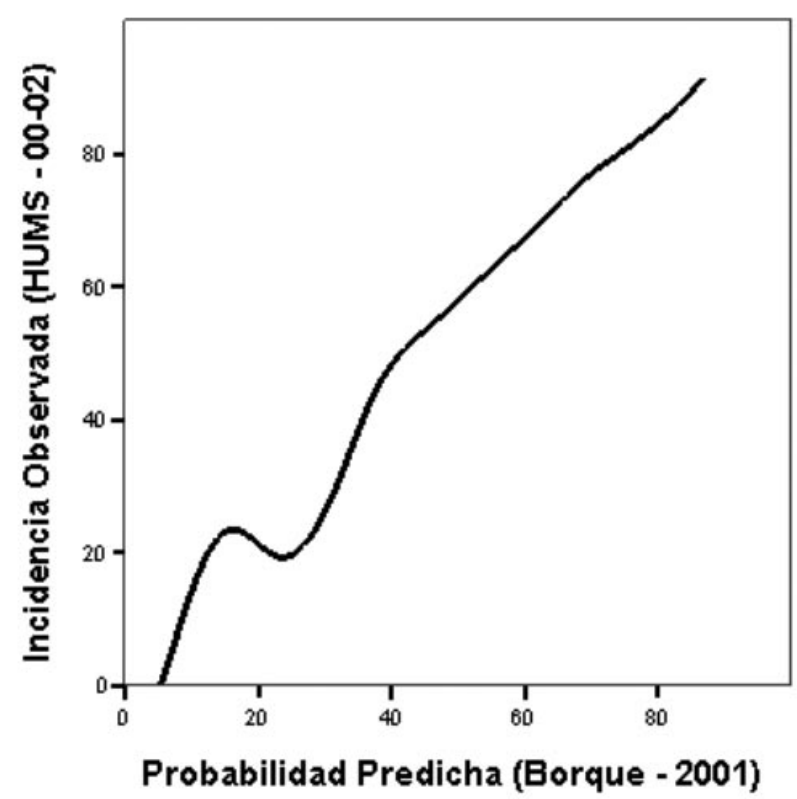

FIGURA 4. Curva de calibración del "NomogramaH.U.M.S." en nuestro medio. a juicio de sus autores (43). En lo referente a la discriminación igualmente es buena cuando se aplica a centros de referencia $(43,45-47)$ en detrimento de cuando se evalúa en estudios multicéntricos $(44,48)$, posiblemente consecuencia de la mejor, más homogénea y fiable determinación de las variables a estudio y su recogida de datos desaconsejando el uso de estos modelos predictivos si no tenemos la suficiente certeza de que las variables predictivas han sido analizadas y recogidas con rigor y por profesionales experimentados. A este respecto se ha llegao incluso a postular la posibilidad de utilizar las "Tablas de Partin" como un método para monitorizar la correcta praxis en este ámbito, de modo que si al aplicarlas a un medio asistencial concreto no demuestran su validez externa ello equivaldría a considerar que las variables predictivas y resultado no se han recogido correctamente aconsejando una revisión de la práctica asistencial en dicho ámbito (50).

Validación de las Tablas de Partin y su confrontación con el nomograma del Hospital Universitario "Miguel Servet" (H.U.M.S.)

En este contexto de validación de las "Tablas de Partin" decidimos en su día validar dicho modelo predictivo en nuestro medio asistencial e incluso compararlo con nuestro propio nomograma.

Para ello escogimos 314 pacientes sometidos a prostatectomía radical entre enero de 2000 y diciembre de 2002, todos ellos posteriores a los pacientes utilizados previamente para generar nuestro modelo y por tanto desconocidos tanto para las "Tablas de Partin" como para el "NomogramaH.U.M.S.".

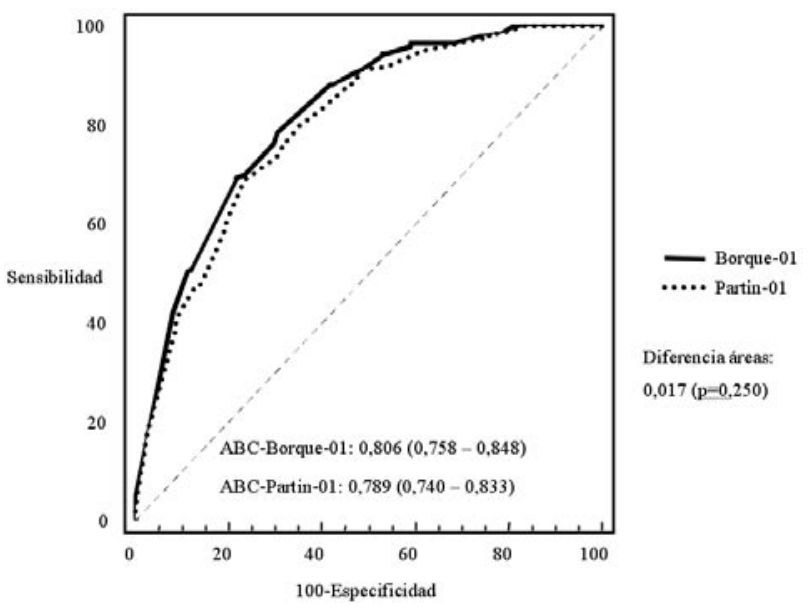

FIGURA 5. Áreas bajo la curva ROC al aplicar ambos modelos a nuestro medio asistencial. 
Las curvas de calibración obtenidas para ambos modelos mostraban una buena correlación con la realidad observada en nuestro medio para ambas predicciones tal y como se muestra en las Figuras 3 y 4

En cuanto a la capacidad de discriminación de ambos modelos en nuestro medio objetivada mediante las áreas bajo la curva ROC es buena para ambos casos si bien quizás algo mejor para nuestro modelo, aunque es cierto que la diferencia de áreas bajo la curva ROC es mínima y sin significación estadística (Figura 5).

$Y$ finalmente en lo que se refiere a la utilidad clínica vemos con respecto a las "Tablas de Partin" que si intuitivamente escogiéramos un punto de corte del $50 \%$, es decir, que decidiéramos que todo aquél paciente que tuviera más de una $50 \%$ de probabilidad de estar órgano-confinado (pT2) realmente lo estuviera anatomo-patológicamente y con ello decidiéramos su mejor opción de tratamiento, se nos escaparía un $24 \%$ de los pacientes que realmente encontramos en nuestro medio órgano-confinados (sensibilidad del 76,21\%) (Tabla II).

Esta cifra no sería admisible a nuestro juicio y de algún modo desearíamos que como mucho nuestro error, nuestra pérdida en la capacidad de identificar a los pacientes órgano-confinados (pT2) y por consiguiente los candidatos ideales a tratamiento de intención curativa, no superase el 10\% o idealmente un $5 \%$. Según apreciamos en la Tabla II, ello nos obligaría a descender nuestro punto de corte a partir del cual considerar como patológicamente órgano-confinado a todo aquél paciente con una probabilidad de pT2 mayor o igual al 35\% (sensibilidad: 91,75\%) o del 30\% (sensibilidad: 96,60\%).

Estas mismas reflexiones con respecto a la utilidad clínica en lo que a nuestro modelo se refiere (Tabla III) el error al considerar este intuitivo $50 \%$ de probabilidad, sería de tan sólo un 12\% (sensibilidad

TABLA II. PREDICCIÓN DE ÓRGANO-CONFINACIÓN SEGÚN PARTIN-O 1.

\begin{tabular}{|c|c|c|c|c|c|c|c|c|c|}
\hline Pto. corte & VP & FN & VN & FP & Precisión & Sensibilidad & Especificidad & VPP & VPN \\
\hline$>10 \%$ & 206 & 0 & 10 & 98 & 68,79 & 100,00 & 9,26 & 67,76 & 100,00 \\
\hline$>15 \%$ & 203 & 3 & 22 & 86 & 71,66 & 98,54 & 20,37 & 70,24 & 88,00 \\
\hline$>20 \%$ & 202 & 4 & 23 & 85 & 71,66 & 98,06 & 21,30 & 70,38 & 85,19 \\
\hline$>25 \%$ & 201 & 5 & 26 & 82 & 72,29 & 97,57 & 24,07 & 71,02 & 83,87 \\
\hline$>30 \%$ & 199 & 7 & 30 & 78 & 72,93 & 96,60 & 27,78 & 71,84 & 81,08 \\
\hline$>35 \%$ & 189 & 17 & 49 & 59 & 75,80 & 91,75 & 45,37 & 76,21 & 74,24 \\
\hline$>40 \%$ & 182 & 24 & 57 & 51 & 76,11 & 88,35 & 52,78 & 78,11 & 70,37 \\
\hline$>45 \%$ & 170 & 36 & 65 & 43 & 74,84 & 82,52 & 60,19 & 79,81 & 64,36 \\
\hline$>50 \%$ & 157 & 49 & 72 & 36 & 72,93 & 76,21 & 66,67 & 81,35 & 59,50 \\
\hline$>55 \%$ & 152 & 54 & 74 & 34 & 71,97 & 73,79 & 68,52 & 81,72 & 57,81 \\
\hline$>60 \%$ & 123 & 83 & 86 & 22 & 66,56 & 59,71 & 79,63 & 84,83 & 50,89 \\
\hline$>65 \%$ & 98 & 108 & 93 & 15 & 60,83 & 47,57 & 86,11 & 86,73 & 46,27 \\
\hline$>70 \%$ & 84 & 122 & 98 & 10 & 57,96 & 40,78 & 90,74 & 89,36 & 44,55 \\
\hline$>75 \%$ & 80 & 126 & 98 & 10 & 56,69 & 38,83 & 90,74 & 88,89 & 43,75 \\
\hline$>80 \%$ & 36 & 170 & 105 & 3 & 44,90 & 17,48 & 97,22 & 92,31 & 38,18 \\
\hline$>85 \%$ & 2 & 204 & 108 & 0 & 35,03 & 0,97 & 100,00 & 100,00 & 34,62 \\
\hline$>90 \%$ & 0 & 206 & 108 & 0 & 34,39 & 0,00 & 100,00 & - & 34,39 \\
\hline & \multicolumn{2}{|c|}{ pT2 $=206$} & \multicolumn{2}{|c|}{$>$ pT2 $=108$} & & & & & \\
\hline
\end{tabular}

(n=314 pacs.: 206 "pT2" vs. 108 "> pT2") 
TABLA III. PREDICCIÓN DE ÓRGANO-CONFINACIÓN SEGÚN BORQUE-O 1.

\begin{tabular}{|c|c|c|c|c|c|c|c|c|c|}
\hline Pto. corte & VP & FN & VN & FP & Precisión & Sensibilidad & Especificidad & VPP & VPN \\
\hline$>10 \%$ & 206 & 0 & 12 & 96 & 69,43 & 100,00 & 11,11 & 98,21 & 100,00 \\
\hline$>15 \%$ & 203 & 3 & 19 & 89 & 70,70 & 98,54 & 17,59 & 69,52 & 86,36 \\
\hline$>20 \%$ & 202 & 4 & 23 & 85 & 71,66 & 98,06 & 21,30 & 70,38 & 85,19 \\
\hline$>25 \%$ & 199 & 7 & 32 & 76 & 73,57 & 96,60 & 29,63 & 72,36 & 82,05 \\
\hline$>30 \%$ & 199 & 7 & 40 & 68 & 76,11 & 96,60 & 37,04 & 74,53 & 85,11 \\
\hline$>35 \%$ & 198 & 8 & 40 & 68 & 75,80 & 96,12 & 37,04 & 74,44 & 83,33 \\
\hline$>40 \%$ & 194 & 12 & 47 & 61 & 76,75 & 94,17 & 43,52 & 76,08 & 79,66 \\
\hline$>45 \%$ & 187 & 19 & 54 & 54 & 76,75 & 90,78 & 50,00 & 77,59 & 73,97 \\
\hline$>50 \%$ & 182 & 24 & 60 & 48 & 77,07 & 88,35 & 55,56 & 79,13 & 71,43 \\
\hline$>55 \%$ & 162 & 44 & 73 & 35 & 74,84 & 78,64 & 67,59 & 82,23 & 62,39 \\
\hline$>60 \%$ & 143 & 63 & 83 & 25 & 71,97 & 69,42 & $23,15 \quad 76,85$ & 85,12 & 56,85 \\
\hline$>65 \%$ & 143 & 63 & 83 & 25 & 71,97 & 69,42 & 76,85 & 85,12 & 56,85 \\
\hline$>70 \%$ & 142 & 64 & 83 & 25 & 71,66 & 68,93 & 76,85 & 85,03 & 56,46 \\
\hline$>75 \%$ & 105 & 101 & 94 & 14 & 63,38 & 50,97 & 87,04 & 88,24 & 48,21 \\
\hline$>80 \%$ & 86 & 120 & 99 & 9 & 58,92 & 41,75 & 91,67 & 90,53 & 45,21 \\
\hline$>85 \%$ & 86 & 120 & 99 & 9 & 58,92 & 41,75 & 91,67 & 90,53 & 45,21 \\
\hline$>90 \%$ & 0 & 206 & 108 & 0 & 34,39 & 0,00 & 100,00 & - & 34,39 \\
\hline & \multicolumn{2}{|c|}{ pT2 $=206$} & \multicolumn{2}{|c|}{$>$ pT2 $=108$} & & & & & \\
\hline
\end{tabular}

(n=314 pacs.: 206 "pT2" vs. 108 "> pT2")

$88,35 \%$ ) que no es sino la mitad del que obteníamos con las "Tablas de Partin".

No obstante aplicando el mismo nivel de exigencia de errar ton sólo en un 10\% o incluso $5 \%$, deberíamos utilizar como punto de corte para considerar un paciente anatomo-patológicamente órgano-confinado, todo aquél al que nuestro modelo considerase "pT2" en igual o más de un $45 \%$ de probabilidad (sensibilidad: $90,78 \%$ ), o un $30 \%$ (sensibilidad: $96,60 \%$ ), respectivamente.

De este modo y de acuerdo a los tres criterios de validación externa reiterados hasta la saciedad en este texto hemos evidenciado que:

- tanto las "Tablas de Partin" como el "modeloH.U.M.S." ofrecen unas probabilidades de órgano-confinación tumoral por cáncer de próstata a partir de factores preoperatorios, que se ajustan bien a la verdadera incidencia de dicha órgano- confianción hallada en nuestro medio (buena calibración);

- su capacidad de separar órgano-confinados de no órgano-confinados en base a dichas predicciones también es buena (discriminación);

- y finalmente que con una buena selección de puntos de corte, quizás algo más próxima al intuitivo $50 \%$ en nuestro modelo, somos capaces de identificar a la inmensa mayoría de los tumores órgano-confinados, siendo posiblemente este aspecto nunca valorado hasta ahora por anteriores validaciones externas de estos modelos predictivos, el que mayor confianza aporta en su uso y el que realmente permitiría su generalización al igual que ocurre con el punto de corte de PSA-total, del porcentaje de PSA-libre/PSA-total para indicar una biopsia, o el valor de PSA-total o Gleason de la biopsia para solicitar una gammagrafía ósea como estudio de diseminación, que no son sino puntos de corte determinados igualmente a par- 
TABLA IV. RESUMEN DE LAS VALIDACIONES PROSPECTIVAS DE LOS NOMOGRAMAS DE PARTIN PUBLICADOS EN 1997 Y 2001, Y DEL NOMOGRAMA DEL H.U.M.S. PUBLICADO EN 2001.

\begin{tabular}{|l|l|c|c|c|}
\hline Validación & Nomograma & CALIBRACIÓN & DISCRIMINACIÓN & UTILIDAD CLÍNICA \\
\hline Blute-00 & Partin -97 & INFRAVALORA & ACEPTABLE & - \\
\hline Penson-02 & Partin -97 & - & MALA & - \\
\hline Graefen-03 & Partin -97 & - & BUENA & - \\
\hline Augustin-04 & Partin -97 & BUENA & BUENA & - \\
\hline Augustin-04 & Partin -01 & BUENA & BUENA & - \\
\hline Steuber-05 & Partin -01 & BUENA & BUENA & - \\
\hline Eskicorapci-05 & Partin -01 & - & MALA & - \\
\hline H.U.M.S.-06 & Partin -01 & BUENA & BUENA & Punto de corte: $>35 \%$ \\
\hline H.U.M.S.-06 & Borque -01 & BUENA & BUENA & Punto de corte: $>45 \%$ \\
\hline
\end{tabular}

tir de valores de sensibilidad, especificidad y valores predictivos como en este caso.

De este modo los nomogramas tras su validación en nuestro medio se confirman como una herramienta más en la ayuda a la toma de decisiones permitiendo por ejemplo en el caso concreto de la estadificación, desde:

- identificar potenciales pacientes de alto riesgo de no órgano-confinación candidatos a pruebas de estadificación de segundo nivel como pudieran ser la biopsia de vesículas seminales, linfadenectomía, tomografía computerizada, resonancia magnética, rastreo óseo, etc ... según la práctica habitual de cada medio,

- seleccionar pacientes con alto riesgo de extracapsularidad y en este sentido pacientes a riesgo de dejar márgenes oncológicos positivos ante una cirugía conservadora de haces neurovasculares,

- o bien facilitar la toma de decisiones terapéuticas seleccionando directamente pacientes para tratamientos de intención curativa, cirugía, radioterapia externa, braquiterapia, crioterapia, ... $\circ$ pacientes candidatos a hormonoterapia concomitante a radioterapia ante alto riesgo de no órgano-confinación.

La realidad es que en nuestro medio tras generar nuestro modelo predictivo con los pacientes intervenidos entre 1986 y 1999, decidimos no aplicarlo hasta validarlo prospectivamente con los pacientes intervenidos entre el año 2000 y el 2002 cuyos resultados aquí hemos expresado.

En el año 2003 comenzó su aplicación como herramienta de ayuda en la toma de decisiones terapéuticas individualizadas para cada paciente tímidamente, y formalmente en los años 2004 y 2005. Con ello, de un porcentaje de infraestadificación clínica que rondaba el 30\% en los años 2000 a 2002 en que no se aplicó y que se sitúa en los rangos de las grandes series actuales de prostatectomía radical, descendimos a un $23,8 \%$ en su primer año de tímida aplicación que en los dos últimos años y merced sin duda a una mejor selección de los pacientes intervenidos se ha situado en un $17,3 \%$ lo que constituye una disminución a casi el $50 \%$ de la infraestadificación clínica que teníamos hace apenas tres años (Figura 6).

Con todo ello y resumiendo las validaciones externas publicadas hasta el momento sobre las "Tablas de Partin" y nuestro propio modelo ofrecemos la siguiente tabla aclaratoria (Tabla IV).

\section{CONCLUSIONES}

En definitiva nuestra propuesta de conclusiones es la siguiente:

1. Que los nomogramas no son sino la simplificación gráfica de complejos análisis multivariante que facilitan su interpretación. 


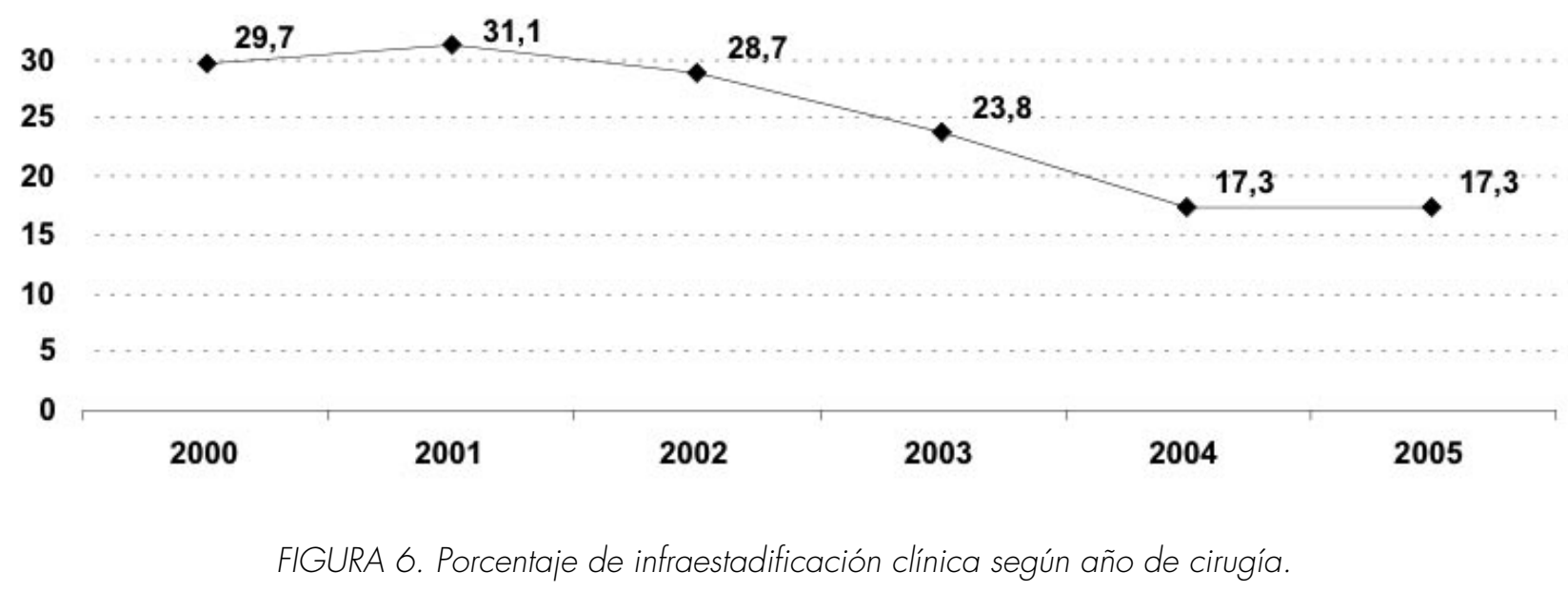

2. Que para poder utilizarlos es indispensable que las variables predictivas en que se fundamentan hayan sido analizadas y recogidas con el más absoluto rigor y fiabilidad.

3. Que previa validación en nuestro medio y sobre todo bien escogidos los puntos de corte para tomar decisiones clínicas, son excelentes herramientas de ayuda en la toma de decisiones para un paciente individual.

4. Que si no demuestran su validez cuando ésta ha sido suficientemente testada en otros medios podría ser pertinente re-evaluar la fiabilidad en el análisis y recogida de las variables predictivas y resultado.

\section{BIBLIOGRAFÍA y LECTURAS RECOMENDADAS ( ${ }^{*}$ lectura de interés $y^{* *}$ lectura fundamental)}

1. DUBOIS, D.; DUBOIS, E.F.: "A formula to estimate the approximate surface area if height and weight be known". Arch. Intern. Medicine, 17: 863, 1916.

**2. BORQUE, A.; SANZ, G.; ALLEPUZ, C. y cols.: "The use of neural networks and logistic regression analysis for predicting pathological stage in men undergoing radical prostatectomy: a population based study". J. Urol., 166: 1672, 2001.

*3. PARTIN, A.W.; KATTAN, M.W.; SUBONG, E.N.P. y cols.: "Combination of prostate-specific antigen, clinical stage, and Gleason score to predict pathological stage of localized prostate cancer”. JAMA, 277: 1445, 1997.
**4. PARTIN, A.W.; MANGOLD, L.A.; LAMM, D.M. y cols.: "Contemporary update of prostate cancer staging nomograms (Partin tables) for the new millennium". Urology, 58: 843, 2001.

*5. KATTAN, M.W.; WHEELER, T.M.; SCARDINO, P.T.: "Postoperative nomogram for disease recurrence after radical prostatectomy for prostate cancer". J. Clin. Oncol., 17: 1499, 1999.

*6. CATAN, M.W.; ZELEFSKY, M.J.; KUPELIAN, P.A. y cols.: "Pretreatment nomogram for predicting the outcome of three-dimensional conformal radiotherapy in prostate cancer". J. Clin. Oncol., 18: 3352, 2000.

*7. KATTAN, M.W.; POTTERS, L.; BLASKO, J.C. y cols.: "Pretreatment nomogram for predicting freedom from recurrence after permanent prostate brachytherapy in prostate cancer". Urology, 58: 393, 2001.

8. KAMOI, K.; BABAIAN, K.J.: "Advances in the application of prostate-specific antigen in the detection of early-stage prostate cancer". Semin. Oncol., 26: 140, 1999.

9. CARLSON, G.D.; CALVANESE, C.B.; PARTIN, A.W.: "An algorithm combining age, total prostate-specific antigen (PSA), and percent free PSA to predict prostate cancer results on 4298 cases". Urology, 52: 455, 1998.

10. EASTHAM, J.A.; MAY, R.; ROBERTSON, J.L. y cols.: "Development of a nomogram that predicts the probability of a positive prostate biopsy in men with an abnormal digital rectal examination and a prostate-specific antigen between 0 and 4 ng/Ml”. Urology, 54: 709, 1999.

11. POTTER, S.R.; HORNIGER, W.; TINZL, M. y cols.: "Age, prostate-specific antigen, and digital rectal examination as determinants of the probability of having prostate cancer". Urology, 57: 1100, 2001. 
12. OHORI, M.; SWINDLE, P.: "Nomograms and instruments for the initial prostate evaluation the ability to estimate the likelihood of identifying prostate cancer". Semin. Urol. Oncol., 20: 116, 2002.

13. DJAVAN, B.; REMZI, M.; ZLOTTA, A. y cols.: "Novel artificial neural network for early detection of prostate cancer". J. Clin. Oncol., 20: 921, 2002.

14. SUZUKI, H.; KOMIYA, A.; KAMIYA, N. y cols.: "Development of a nomogram to predict probability of positive initial prostate biopsy among Japanese patients". Urology, 67: 131, 2006.

15. KARAKIEWICZ, P.I.; BENAYOUN, S.; KATTAN, M.W. y cols.: "Development and validation of a nomogram predicting the outcome of prostate biopsy based on patient age, digital rectal examination and serum prostate specific antigen". J. Urol., 173: 1930, 2005.

16. YANKE, B.V.; GONEN, M.; SCARDINO, P.T. y cols.: "Validation of a nomogram for predicting positive repeat biopsy for prostate cancer". J. Urol., 173: 421, 2005.

17. REMZI, M.; FONG, Y.K.; DOBROVITS, M. y cols.: "The Vienna nomogram: validation of a novel biopsy strategy defining the optimal number of cores based on patient age and total prostate volume". J. Urol., 174: 1256, 2005.

*18. ROSS, P.L.; SCARDINO, P.T.; KATTAN, M.W.: "A catalog of prostate cancer nomograms". J. Urol., 165: 1562, 2001.

19. OHORI, M.; KATTAN, M.W.; KOH, H. y cols.: "Predicting the presence and side of extracapsular extension: a nomogram for staging prostate cancer". J. Urol., 171: 1844, 2004.

20. CAGIANNOS, I.; KARAKIEWICZ, P.; EASTHAM, J.A. y cols.: "A preoperative nomogram identifying decreased risk of positive pelvic lymph nodes in patients with prostate cancer". J. Urol., 170: 1798, 2003.

21. KATTAN, M.W.; EASTHAM, J.A.; WHEELER, T.M. y cols.: "Counseling men with prostate cancer: a nomogram for predicting the presence of small, moderately differentiated, confined tumors". J. Urol., 170: 1792, 2003.

22. KOH, H.; KATTAN, M.W.; SCARDINO, P.T. y cols.: "A nomogram to predict seminal vesicle invasion by the extent and location of cancer in systematic biopsy results". J. Urol., 170: 1203, 2003.

23. KATTAN, M.W.; EASTHAM, J.A.; STAPLETON, A.M. y cols.: "A preoperative nomogram for disease recurrence following radical prostatectomy for prostate cancer". J. Natl. Cancer Inst., 90: 766, 1998.

24. D'AMICO, A.V.; WHITTINGTON, R.; MALKOWICZ, S.B. y cols.: "The combination of preoperative prostate specific antigen and posto- perative pathological findings to predict prostate specific antigen outcome in clinically localized prostate cancer". J. Urol., 160: 2096, 1998.

25. D'AMICO, A.V.; WHITTINGTON, R.; MALKOWICZ, S.B. y cols.: "Combination of the preoperative PSA level, biopsy gleason score, percentage of positive biopsies, and MRI T-stage to predict early PSA failure in men with clinically localized prostate cancer". Urology, 55: 572, 2000.

26. HERMAN, C.M.; WILCOX, G.E.; KATTAN, M.W. y cols.: "Lymphovascular invasion as a predictor of disease progression in prostate cancer". Am. J. Surg. Pathol., 24: 859, 2000.

27. PALISAAR, R.J.; GRAEFEN, M.; KARAKIEWICZ, P.I. y cols.: "Assessment of clinical and pathologic characteristics predisposing to disease recurrence following radical prostatectomy in men with pathologically organ-confined prostate cancer". Eur. Urol., 41: 155, 2002.

28. HAN, M.; PARTIN, A.W.; ZAHURAK, M. y cols.: "Biochemical (prostate specific antigen) recurrence probability following radical prostatectomy for clinically localized prostate cancer". J. Urol., 169: 517, 2003.

29. KATTAN, M.W.; SHARIAT, S.F.; ANDREWS, B. y cols.: "The addition of interleukin-6 soluble receptor and transforming growth factor beta1 improves a preoperative nomogram for predicting biochemical progression in patients with clinically localized prostate cancer". J. Clin. Oncol., 21: 3573, 2003.

30. KHAN, M.A.; PARTIN, A.W.; MANGOLD, L.A. y cols.: "Probability of biochemical recurrence by analysis of pathologic stage, Gleason score, and margin status for localized prostate cancer". Urology, 62: 866, 2003.

31. SAN FRANCISCO, I.F.; REGAN, M.M.; OLUMI, A.F. y cols.: "Percent of cores positive for cancer is a better preoperative predictor of cancer recurrence after radical prostatectomy than prostate specific antigen". J. Urol., 171: 1492, 2004.

32. POULAKIS, V.; WITZSCH, U.; DE VRIES, R. y cols.: "Preoperative neural network using combined magnetic resonance imaging variables, prostate specific antigen, and Gleason score to predict prostate cancer recurrence after radical prostatectomy". Eur. Urol., 46: 571, 2004.

33. DOTAN, Z.A.; BIANCO, F.J. Jr.; RABBANI, F. y cols.: "Pattern of prostate-specific antigen (PSA) failure dictates the probability of a positive bone scan in patients with an increasing PSA after radical prostatectomy". J. Clin. Oncol., 23: 1962, 2005.

34. STEPHENSON, A.J.; SCARDINO, P.T.; EASTHAM, J.A. y cols.: "Postoperative nomogram predicting the 10 -year probability of prostate 
cancer recurrence after radical prostatectomy". J. Clin. Oncol., 23: 7005, 2005.

35. STROM, S.S.; WANG, X.; PETTAWAY, C.A. y cols.: "Obesity, weight gain, and risk of biochemical failure among prostate cancer patients following prostatectomy". Clin. Cancer Res., 11: 6889, 2005.

36. D'AMICO, A.V.; WHITTINGTON, R.; MALKOWICZ, S.B. y cols.: "Pretreatment nomogram for prostate-specific antigen recurrence after radical prostatectomy or external-beam radiation therapy for clinically localized prostate cancer". J. Clin. Oncol., 17: 168, 1999.

37. PARKER, C.C.; NORMAN, A.R.; HUDDART, R.A. y cols.: "Pre-treatment nomogram for biochemical control after neoadjuvant androgen deprivation and radical radiotherapy for clinically localised prostate cancer". Br. J. Cancer, 86: 686, 2002.

38. KATTAN, M.W.;ZELEFSKY, M.J.; KUPELIAN, P.A. y cols.: "Pretreatment nomogram that predicts 5-year probability of metastasis following three-dimensional conformal radiation therapy for localized prostate cancer". J. Clin. Oncol., 21: 4568, 2003.

39. SMALETZ, O.; SCHER, H.I.; SMALL, E.J. y cols.: "Nomogram for overall survival of patients with progressive metastatic prostate cancer after castration”. J. Clin. Oncol., 20: 3972, 2002.

40. SVATEK, R.; KARAKIEWICZ, P.I.; SHULMAN, M. y cols.: "Pre-treatment nomogram for disease-specific survival of patients with chemotherapy-naive androgen independent prostate cancer". Eur. Urol., 49: 666, 2006.

**41. VERGOUWE, Y.; STEYERBERG, E.W.; EIJKEMANS, M.J. y cols.: "Validity of prognostic models: when is a model clinically useful?". Semin. Urol. Oncol., 20: 96, 2002.

**42. DI BLASIO, C.J.; RHEE, A.C.; CHO, D. y cols.: "Predicting clinical end points: treatment nomograms in prostate cancer". Semin. Oncol., 30: $567,2003$.
*43. BLUTE, M.L.; BERGSTRALH, E.J.; PARTIN, A.W. y cols.: "Validation of Partin tables for predicting pathological stage of clinically localized prostate cancer". J. Urol., 164: 1591, 2000.

*44. PENSON, D.F.; GROSSFELD, G.D.; LI, Y.P. y cols.: "How well does the Partin nomogram predict pathological stage after radical prostatectomy in a community based population? Results of the cancer of the prostate strategic urological research endeavour". J. Urol., 167: 1653, 2002.

*45. GRAEFEN, M.; AUGUSTIN, H.; KARAKIEWICZ, P.I. y cols.: "Can predictive models for prostate cancer patients derived in the United States of America be utilized in European patients? A validation study of the Partin tables". Eur. Urol., 43: 6, 2003.

*46. AUGUSTIN, H.; EGGERT, T.; WENSKE, S. y cols.: "Comparison of accuracy between the Partin tables of 1997 and 2001 to predict final pathological stage in clinically localized prostate cancer". J. Urol., 171: 177, 2004.

*47. STEUBER, T.; KARAKIEWICZ, P.I.; AUGUSTIN, H. y cols.: "Transition zone cancers undermine the predictive accuracy of Partin table stage predictions". J. Urol., 173: 737, 2005.

*48. ESKICORAPCI, S.Y.; KARABULUT, E.; TURKERI, L. y cols.: "Validation of 2001 Partin tables in Turkey: a multicenter study". Eur. Urol., 47: 185, 2005.

49. BORQUE, A.: "Validación del nomograma de Partin frente al nomograma del Hospital Universitario Miguel Servet”. Presentación oral presentada en la mesa redonda sobre Cáncer de próstata. Curso Internacional de Urología,Zaragoza, Febrero 2006.

**50. KELLEHER, F.C.; ARMSTRONG, J.: “A method for assessing accurate application of the Partin Tables in the pre-therapy evaluation of patients with prostate cancer". Clin. Oncol. (R. Coll. Radiol.), 17: 659, 2005. 